\title{
Serum Concentration of Myelin Basic Protein as a Prognostic Marker in Mild-to-moderate Head Injury Patients: A Prospective Study in a Tertiary Care Center
}

\author{
Ashvamedh Singh ${ }^{1, \odot} \quad$ Kulwant Singh ${ }^{1} \quad$ Anurag Sahu ${ }^{1, \odot} \quad$ R.S. Prasad ${ }^{1} \quad$ N. Pandey ${ }^{1} \quad$ Sambuddha Dhar ${ }^{1}$ \\ ${ }^{1}$ Department of Neurosurgery, Institute Of Medical Sciences (IMS), \\ Address for correspondence Anurag Sahu, MCh, Department of \\ Banaras Hindu University, Varanasi, Uttar Pradesh, India \\ Neurosurgery, IMS-BHU, Varanasi 221005, Uttar Pradesh, India \\ (e-mail: anuragsahu25@rediffmail.com).
}

Indian J Neurosurg 2022;11:216-220

\begin{abstract}
Objective To estimate the level of myelin basic protein (MBP) and look for its validity in outcome prediction among mild-to-moderate head injury patients.

Materials and Methods It was a prospective study done at the Department of Neurosurgery, Institute of Medical Sciences, Banaras Hindu University from Jan 2018 to July 2019. All patients who presented to us within 48 hours of injury with mild-to-moderate head injury with apparently normal CT brain were include in the study. The serum sample were collected on the day of admission and 48 hours later, and patients were treated with standard protocols and observed 6 months postdischarge. Results Of the 32 patients enrolled, we observed mean MBP level was higher for severity of brain damage, but not associated with age, mode of injury, and radiological diag-

Keywords

- MBP

- serum biomarker

- myelin basic protein

- traumatic brain injury

- serum biomarker in TBI nosis. Mean MBP levels were not statistically associated with Glasgow coma scale (GCS) score at admission but was correlated to outcome with $p<0.05$, with sensitivity of $50 \%$ and specificity $72 \%$, that is, patients with good outcome have lower mean MBP levels.

Conclusion MBP as per our analysis can be used as a prognostic marker in patients with head injury. It is not the absolute value rather a trend showing rise in serum MBP levels, which carries a significant value in outcome prediction.
\end{abstract}

\section{Introduction}

Traumatic brain injury (TBI), a major public health problem, results in deaths, injuries and disabilities in all age groups. The major cause being economic, industrial advancements and increased motorization combined with the pleasure of thrill and adventure among youngsters. ${ }^{1}$ It is observed more in young and productive persons and more so in males. India has a huge population, with quarter of the world's trauma deaths occurring here. ${ }^{2,3}$ Although national-level data is not available for TBI, but it is believed that in India over a million deaths are due to trauma and roughly $50 \%$ are because of TBI. Road traffic accidents (RTA) is a major cause of TBI (60\%), followed by falls $25 \%$ and assaults $10 \%{ }^{1}$

DOI https://doi.org/ $10.1055 / \mathrm{s}-0040-1716936$ ISSN 2277-954X
TBI currently can be classified using the Glasgow coma scale (GCS) score into mild, moderate, or severe. Mild head injury constitutes roughly 60 to 70 percent of all cases of TBI, and majority amongst them will go on to have some form of behavioral and cognitive symptoms, which are referred to as postconcussive syndrome (PCS). ${ }^{4}$ Since diffuse axonal and small vessel injury in the brain contribute presumptively to PCS, that injury per se is not typically detected by CT. Several studies have recently assessed and shown the relation between brain-related proteins found in the serum and the acute and delayed mild TBI outcomes in victims of closed head injury. There is a leakage of protein into extracellular matrix poststructural damage to the neuronal and

\footnotetext{
(c) 2022. Neurological Surgeons' Society of India.

This is an open access article published by Thieme under the terms of the Creative Commons Attribution-NonDerivative-NonCommercial-License, permitting copying and reproduction so long as the original work is given appropriate credit. Contents may not be used for commercial purposes, or adapted, remixed, transformed or built upon. (https://creativecommons.org/licenses/by-nc-nd/4.0/)

Thieme Medical and Scientific Publishers Pvt. Ltd., A-12, 2nd Floor, Sector 2, Noida-201301 UP, India
} 
supporting cells. So if the blood-brain barrier is damaged, these proteins may then enter the peripheral circulation, where they can be sampled. ${ }^{5}$ Myelin basic protein (MBP) is one such marker component of oligodendrocytes, the second most abundant protein in the central nervous system (CNS). It gets released into circulation following axonal injury. The levels are raised in 24 to 48 hours after TBI and persist for 2 weeks or so, making it a desirable marker for prognostication and also screening among children with mild TBI. ${ }^{6-8}$

Majority of patient's management and prognosis is based on GCS score and imaging studies are also of little help in prognosticating, so there is a need for a potential biomarker that provides us with the maximum information regarding the short-term and long-term outcomes. The study was done to see if MBP can serve as a potential biomarker.

\section{Materials and Methods}

The prospective study was conducted in the Department of Neurosurgery, Institute of Medical Sciences, Banaras Hindu University, Varanasi from January 2018 to July 2019.

Inclusion criteria:

- All age groups.

- Mild and moderate head injury.

- Closed head injury with apparent CT brain normal.

- No hematological disorder at time of admission.

- Family or next-of-kin available to provide written informed consent.

\section{Exclusion criteria:}

- Patients with neurologic disabilities (head injury, cerebral infarction, and hemorrhage) prior to the injury.

- Patients presenting at the emergency more than 48 hours after injury.

- Prolonged cardiac arrest at the scene of the accident, or high cervical spinal cord injury, and those who had died from uncontrollable hemorrhage or multiple life-threatening associated injuries.

- Pregnancy and lactating mother.

- Patients with known coagulopathy or clotting disorder or patient on antiplatelets.

\section{Study Protocol}

Patients were selected randomly with the help of the envelope method. Those meeting the required criteria were included and observed. Patient's demographic information was recorded at the time of enrollment.

Neurological examination was reformed on regular intervals and finding of GCS score and pupillary status were noted at admission and subsequently at 48 hours.

Mode of injury, associated injuries, time of patient arrival at the hospital emergency, cranial CT scan findings, days of hospital stay, and outcome on basis of GCS score were judged and recorded.

Serum sample collection was done on the day of admission and 48 hours later. The method of obtaining serum was as follows:
Nonpyrogenic was used to avoid any cell stimulation, and blood sample was collected and centrifuged at $3000 \mathrm{rpm}$ for 10 minutes. Carefully, serum was separated from red blood cells as quickly as possible. If precipitation appeared, the sample was centrifuged again.

After collection, the sample was stored at 2 to 8 degrees to be used later at test date.

For estimating the quantity of serum MBP, we used the human MBP autoantibody (MBP-Ab) enzyme-linked immunosorbent assay (ELISA) kit provided by QAYEE BIO for life science. The kit uses a double antibody sandwich onestep assay process (ELISA) to assay the level of MBP-Ab in samples.

Assay method: The kit uses a double antibody sandwich one-step assay process (ELISA), which is contrary to traditional ELISA methods, as only a single incubation and wash step is required.

1. The plate has $8 \times 12$ strips equaling 96 wells. Every sample was made according to our requirement.

2. Set blank wells, standard wells, and test sample wells. respectively:

(1) Blank well: we did not add samples and horseradish peroxidase (HRP); other operations were the same.

(2) Standard wells: added standard $50 \mu \mathrm{L}$ to standard wells.

(3) Test sample wells: Added $40 \mu \mathrm{L}$ of special diluent and then added $10 \mu \mathrm{L}$ of sample. (The final sample dilution is five times and the final result calculation should be multiplied by five times).

(4) Added $50 \mu \mathrm{L}$ of HRP into each well, except the blank well. Thereafter, we sealed the plate, gently shook, and incubated for 60 minutes at $37^{\circ} \mathrm{C}$.

3. Discarded liquid excess, filled each well with diluted washing liquid, mixed and shook for 30 seconds, discarded the washing liquid. and tapped the plate into absorbent papers to dry. Repeated five times and then patted dry.

4. Added $50 \mu \mathrm{L}$ of chromogen solution A to each well, and then added $50 \mu \mathrm{L}$ of chromogen solution $B$ to each well. Gently shook and incubated for 10 minutes at $37^{\circ} \mathrm{C}$ away from light.

5. Stop: added stop solution $50 \mu \mathrm{L}$ into each well to stop the reaction (the blue changes into yellow immediately).

6. Final measurement: Set blank well zero and measured the optical density (OD) at $450 \mathrm{~nm}$ wavelength, which was performed within 15 minutes after adding the stop solution.

According to standards' concentration and the corresponding OD values, calculated the standard curve linear regression equation, and then applied the OD values of the sample onto the regression equation to calculate the corresponding sample's concentration.

The entire patient's data was filled in the predefined pro forma and relevant statistical analysis was performed using SPSS software and nonparametric and parametric tests.

Patients were managed as per institutional guidelines and followed-up for 6 months for neurological outcome, PCS. 
Patients were called for follow-up at 6 months for assessment and those who did not report were contacted telephonically

\section{Results}

Of the total 32 patients who were enrolled in the study, the majority were male $21(65.6 \%)$ and rest female $11(34.4 \%)$ and maximum representation was from those who were in their third decade of life with mean age being 28.48 years (median 25.50 years, range $8-80$ years.).

RTA was the main causative factor responsible for injury $84.37 \%$ and assault was responsible for $15.64 \%$ of cases.

All patients presented within 48 hours of injury, with mean 19.75 hours (median 17 hours, range 3-44 hours).

Loss of consciousness (LOC) was the main presenting complaint amongst patients, mean duration of LOC was 25.03 minutes (median 15 minutes, range of 2-120 minutes). Vomiting was present in $68.8 \%$ (mean five episodes, median three episodes and range of 2-12 episodes) and seizure was present in $18.8 \%$ of patients.

GCS score at admission was noted, with mean being 12.09 (range 9-15, median 11) which at 48 hours postadmission changed to 13.65 (median 15, range 9-15). Of the total patients, 14 (43.8\%) patients had a GCS score of 13 to 15 at admission and 18 (56.3\%) had 9 to 12 at admission (-Fig. 1).

The mean MBP values at admission deciphered by our tests were $107.27 \mathrm{ng} / \mathrm{mL}$ with $\mathrm{SD}$ of $73.51 \mathrm{ng} / \mathrm{mL}$, and the mean MBP value observes at 48 hours postadmission was $65.82 \mathrm{ng} / \mathrm{mL}$ with SD $34.45 \mathrm{ng} / \mathrm{mL}$.

On analysis, it was seen that the mean MBP increased with age of the patient, but it was not statistically significant. The values of mean MBP were more elevated for female gender $153.91 \mathrm{ng} / \mathrm{mL}$ with SD of $73.69 \mathrm{ng} / \mathrm{mL}$ as compared with $82.84 \mathrm{ng} / \mathrm{mL}$ with SD of $61.96 \mathrm{ng} / \mathrm{mL}$ for males but no significance could be deciphered out of it.

Mean MBP bore no relation to mode of injury or symptoms at presentation like LOC, vomiting and seizure, pupillary status at admission, age, gender, mode of injury. Symptoms at presentation do not have any association with outcome of patient.

It was seen that mean MBP levels were higher for patient with GCS scores in the range of 9 to12 (112.40 ng/ $\mathrm{mL}+/-65.81)$ versus 13 to $15(103.28 \mathrm{ng} / \mathrm{mL}+/-80.65)$ at

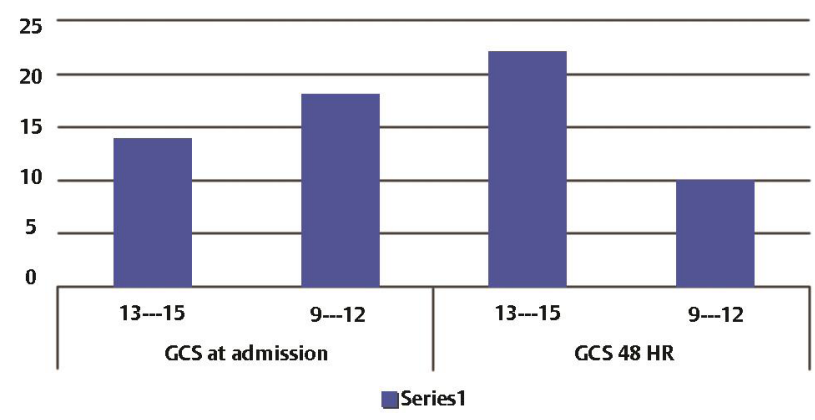

Fig. 1 Glasgow coma scale (GCS) score at admission and 48 hours. admission, that is, with severity of injury, mean MBP levels rise (-Fig. 2).

At 48 hours postadmission, it was seen that the patients who still had their GCS score in the range of 9 to 12 had more mean MBP (85.31 ng/ mL) levels compared with the 13 to 15 group (56.95 ng/ mL) and this was of statistical significance $p=0.011$ (-Fig. 2).

Since the GCS at admission is considered the gold standard for the assessment of injury severity and prediction outcome, we compared the sensitivity and specificity of the GCS score to that of the biomarker trajectory for predicting poor outcome. To maximize specificity (e.g., possibility that a subject predicted to have a poor outcome would have a poor outcome), we assumed that a GCS score of 9 to 12 would predict poor outcome, while GCS scores of 13 to 15 would predict good outcome. Using this assumption, the sensitivity and specificity of the GCS score for predicting poor outcome was $90 \%$ and $59.09 \%$, respectively.

Based on the at admission and 48 hours MBP values, we divided the patients into risers and decliners and assessed the sensitivity and specificity of MBP in predicting outcome: sensitivity $50 \%$ and specificity $72 \%$ respectively (-Table $\mathbf{1}$ ).

When mean MBP levels were compared with outcome of patient, it was seen that patients with good outcome had lower mean MBP levels at 48 hours, which was found to be statistically significant $p=0.011$.

Patients were followed-up for 6 months to look for any symptoms of PCS and it was seen to affect $32.3 \%$ of our patients. When this data was analyzed with MBP levels

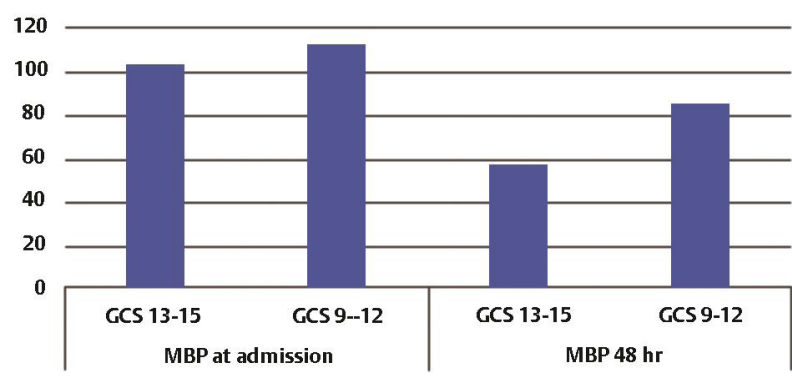

Fig. 2 Myelin basic protein (MBP) at admission and 48 hours.

Table 1 GCS predicting outcome

\begin{tabular}{|l|l|l|}
\hline GCS & Poor outcome & Good outcome \\
\hline $13-15$ & 9 & 9 \\
\hline $9-12$ & 1 & 13 \\
\hline & \multicolumn{2}{|l|}{ Sensitivity $9 / 10=90 \%$, specificity $3 / 22=59 \%$} \\
\hline & MBP predicting outcome & \multicolumn{1}{l|}{} \\
\hline MBP trajectory & Poor outcome & Good outcome \\
\hline Risers & 5 & 6 \\
\hline Decliners & 5 & 16 \\
\hline & Sensitivity $5 / 10=50 \%$, specificity $6 / 22=72 \%$ \\
\hline
\end{tabular}

Abbreviations: GCS, Glasgow coma scale; MBP, myelin basic protein. 
during admission postinjury, it was seen that values of mean MBP were higher in the patients who developed PCS but no statistical significance could be drawn.

\section{Discussion}

TBI poses the substantial burden of disability and death occurring in low- and middle-income countries. In studies by Gururaj et $\mathrm{al}^{9}$ and Nath et $\mathrm{al}^{10}{ }^{10}$ the majority of TBI patients were in their 3rd decade with a male preponderance; likewise in our study, we had a male:female ratio of 1.9:1 and maximum patients in their 3rd decade.

RTA constitutes the main cause of TBI, possibly because of urbanization and industrialization; moreover, youngsters take pride in living a high adrenaline rush life, driving rash and fast. The data was consistent with studies done in the past by Saxena et $\mathrm{al}^{11}$ and Nath et $\mathrm{al}^{10} \mathrm{TBI}$ is more difficult to assess. The clinical examination is of restricted value during the first hours and days after a head injury. Today, most of the diagnostic process is based on modern neuroimaging techniques, such as CT scanning, MRI, and single-photon emission CT scanning. CT scanning has a relatively low sensitivity for diffuse brain damage, and the availability of MRI is limited; hands get tied in cases with apparently normal CT scan findings, thereby drawing interest toward serum biomarkers as a tool in prognostication.

Majority of TBI in our study came from RTA which holds like the previous studies in TBI, due to rise of urbanization, increased transportation and industrialization. ${ }^{1}$

After head injury, as the severity of TBI increases, bloodbrain barrier is breached, allowing MBP to enter circulation, Thus, increased MBP concentration was found in moderate head injury as compared with mild head injury with values remaining high for days, indicating continuing release of $\mathrm{MBP}^{7,8,12}$

In study by Thomas et al, it was observed that patients with increasing severity of injury and poorer outcome had a higher mean MBP level, that is, patients who died had higher levels of MBP than severely disabled, moderately injured and controls, proving the role of MBP in prognostication. ${ }^{7}$ Similarly, Noseworthy et al, who studied cerebrospinal fluid (CSF) MBP, stated that MBP level correlated with GCS score at day 7, 3 months, 6 months $(p<0.05)$ postinjury, concluding it to be a prognostic marker in assessing outcome. $^{8}$

Yamazaki et al ${ }^{13}$ also analyzed that mean MBP level was $1.4+/-1.5 \mathrm{ng} / \mathrm{mL}$ in survivors and $11.3+/-9.5 \mathrm{ng} / \mathrm{mL}$ in dead cases, indicating a poorer outcome with higher levels. ${ }^{9}$

A study by Su et al showed that mean MBP level showed a sustained rise post TBI compared with controls till day 5 without any overlap; sustained release may be due to myelin breakdown because of secondary injury or Wallerian degeneration. ${ }^{6}$ Also, levels were maximum between day 2 to day 6 in patients with poorer outcome. . $^{6-8}$

In our study, mean MBP levels at 48 hours postadmission correlated to outcome of patient, that is, more the MBP, more severe the injury and poorer the outcome, roughly making the sampling between 2 to 6 days of injury being the best time to sample a patient when MBP was used as a prognostic index marker, which was consistent with the previous study by Su et al and Thomas et al. ${ }^{6-9}$

The trajectory analysis is a type of analysis which can capture variance in biomarker concentrations over time and has been used with success in the social sciences. In study by Berger et al in $2011,{ }^{14,15}$ trajectory analysis of serum biomarker concentrations facilitates outcome prediction after pediatric traumatic and hypoxemic brain injury. They used trajectory analysis to evaluate the ability of the serum concentrations of 3 brain-specific biomarkers-S100B, neuron-specific enolase (NSE) and myelin basic protein (MBP)-to predict poor outcome (Glasgow outcome scale [GOS] scores 3-5) after pediatric TBI.For MBP, the 3-group model predicted poor outcome with a sensitivity of $73 \%$ and specificity of $61 \%$. Thus, when the models predicted a poor outcome, there was a very high probability of a poor outcome. ${ }^{15}$ In our study, the trajectory analysis for MBP (sensitivity $50 \%$ and specificity $72 \%$ ) provided much more specificity for the prediction of poor outcome than the current gold standard, the GCS score (sensitivity $90 \%$ and specificity $59.09 \%$ ).

\section{Conclusion}

Currently, there are no single reliable biochemical markers which indicate degree of brain damage and prognosis after head injury in patients. MBP is a potential biomarker which can be used as a prognostic marker in cases of mild and moderate head injury patients with apparently normal CT brain. Measurement of serum MBP. may be a useful biochemical complement to clinical assessment. It can grade severity of injury and also predict outcome of the patient. Second, it is the rise of level MBP rather than a single value which is of more value, indicating possibility of ongoing damage due to secondary brain injury or Wallerian degeneration, thus predicting outcome of the patient.

Its use as a screening tool and diagnosis is mentioned in literature in pediatric patients. The same cannot be said based on our study, because of paucity of pediatric cases. Further studies with larger sample size and inclusion of severe TBI patients may be required to forego its shortcoming as a prognostic marker and validate our results. Further work is required to evaluate, in individual patients, relationships between serum MBP profiles, clinical assessment in the acute phase after head injury, and long-term follow-up.

\section{Funding}

None.

\section{Conflict of Interest}

None declared.

\section{Acknowledgment}

The authors are thankful to Dr. Gopal Nath, Professor Department of Microbiology IMS BHU and his laboratory staff as well as Mr. Rajesh for his patience and the time he made available to us for the ELISA testing in his laboratory. 


\section{References}

1 Agrawal A, Munivenkatappa A, Shukla DP, et al. Traumatic brain injury related research in India: An overview of published literature. Int J Crit Illn Inj Sci 2016;6(2):65-69

2 Maas AI. Traumatic brain injury in India: a big problem in need of data. Neurol India 2017;65(2):257-258

3 Bogoslovsky T, Gill J, Jeromin A, Davis C, Diaz AR. Fluid biomarkers of traumatic brain injury and intended context of use. Diagnostics (Basel) 2016;6(4):37

4 Levin HS, Diaz-Arrastia RR. Diagnosis, prognosis, and clinical management of mild traumatic brain injury. Lancet Neurol 2015;14(5):506-517

$5 \mathrm{Kim} \mathrm{HJ}$, Tsao JW, Stanfill AG. The current state of biomarkers of mild traumatic brain injury. JCI Insight 2018;3(1):e97105

6 Su E, Bell MJ, Kochanek PM, et al. Increased CSF concentrations of myelin basic protein after TBI in infants and children: absence of significant effect of therapeutic hypothermia. Neurocrit Care 2012;17(3):401-407

7 Thomas DG, Palfreyman JW, Ratcliffe JG. Serum-myelinbasic-protein assay in diagnosis and prognosis of patients with head injury. Lancet 1978;1(8056):113-115

8 Noseworthy TW, Anderson BJ, Noseworthy AF, et al. Cerebrospinal fluid myelin basic protein as a prognostic marker in patients with head injury. Crit Care Med 1985;13(9):

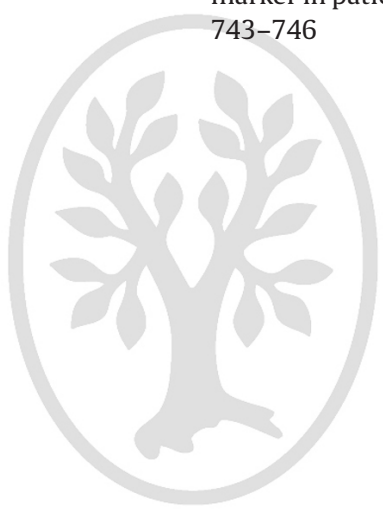

9 Gururaj G. Epidemiology of traumatic brain injuries: Indian scenario. Neurol Res 2002;24(1):24-28

10 Nath HD, Tandon V, Mahapatra AK, et al. Outcome of head injury in unknown patients at level 1 apex trauma centre. IJNT 2011;8(1):11-16

11 Saxena MK, Saddichha S, Pandey V, et al. Prehospital determinants of outcome in traumatic brain injury:Experience from first comprehensive integrated prehospital care providers in India. GVK-EMRI Experience. IJNT 2010;7(2):129-134

12 Mao $\mathrm{Q}$ Chen J, Li N, et al. [The value of serum myelin basic protein in assessment of severity of acute closed head trauma]. Hua Xi Yi Ke Da Xue Xue Bao 1995;26(2):135-137

13 Yamazaki Y, Yada K, Morii S, Kitahara T, Ohwada T. Diagnostic significance of serum neuron-specific enolase and myelin basic protein assay in patients with acute head injury. Surg Neurol 1995;43(3):267-270, discussion 270-271

14 Berger RP, Beers SR, Richichi R, Wiesman D, Adelson PD. Serum biomarker concentrations and outcome after pediatric traumatic brain injury. J Neurotrauma 2007;24(12):1793-1801

15 Berger RP, Bazaco MC, Wagner AK, Kochanek PM, Fabio A. Trajectory analysis of serum biomarker concentrations facilitates outcome prediction after pediatric traumatic and hypoxemic brain injury. Dev Neurosci 2010;32(5-6):396-405 